11

\title{
ИК спектроскопия бидистиллированной и дейтериевой воды в условиях геометрического ограничения в нанопорах стекла
}

\author{
(C) А.В. Быков, Ю.В. Старокуров, А.М. Салецкий \\ Московский государственный университет им. М.В. Ломоносова, фризический фракультет, \\ 119991 Москва, Россия \\ e-mail:sam@physics.msu.ru
}

Поступила в редакцию 23.08.2019 г.

В окончательной редакции 23.08.2019 г.

Принята к публикации 26.09.2019 г.

Проведено исследование ИК спектров $\mathrm{H}_{2} \mathrm{O}$ и $\mathrm{D}_{2} \mathrm{O}$ в условиях геометрического ограничения в матрицах нанопористого стекла (ПС) с пор различных размеров. Установлено, что с увеличением размера пор ПС в области $2<R<6 \mathrm{~nm}$ происходит уменьшение доли воды с сильной Н-связью и увеличение доли воды со слабой Н-связью молекул. При $R>6 \mathrm{~nm}$ изменение в структуре геометрически ограниченных $\mathrm{H}_{2} \mathrm{O}$ и $\mathrm{D}_{2} \mathrm{O}$ не наблюдается. При этом для $\mathrm{D}_{2} \mathrm{O}$ этот эффект меньше (из-за более сильной связи $\mathrm{D}-\mathrm{O}$ по сравнению с $\mathrm{H}-\mathrm{O}$ ). Показано, что молекулы $\mathrm{H}_{2} \mathrm{O}$, которые образуют связи с поверхностью пор, в основном формируются из молекул сетки водородных связей, колебания которых происходят не синфазно, в то время как для $\mathrm{D}_{2} \mathrm{O}$ такие связи с поверхностью формируются из молекул сетки водородных связей, колебания которых происходят синфазно.

Ключевые слова: бистиллированная вода, дейтериевая вода, нанопористое стекло, ИК спектроскопия, водородные связи.

DOI: $10.21883 /$ OS.2020.01.48847.247-19

\section{Введение}

Вода, являющаяся универсальным растворителем и важным компонентом биосферы, играет важнейшую роль во всех известных геологических и биологических процессах. Физико-химические свойства воды контролируются сетью водородных связей (Н-связей), которая состоит из межмолекулярных взаимодействий ее молекул. Эти взаимодействия (Н-связи) являются динамическими и благодаря молекулярной форме и дипольному моменту воды каждая молекула может образовывать до четырех прямых Н-связей [1].

Особый интерес для биосферы представляет дейтериевая вода $\left(\mathrm{D}_{2} \mathrm{O}\right)$, которая применяется в качестве изотопного индикатора в химии и биологии. Тяжелая вода оказывает замедляющее действие на протекание физикохимических процессов в животных и растительных организмах. Способность воды отдавать и принимать водородные связи приводит к уникальным и сложным коллективным динамическим явлениям, связанным с ее сетью водородных связей. Такая способность различна для $\mathrm{D}_{2} \mathrm{O}$ и $\mathrm{H}_{2} \mathrm{O}$. Так, в [2] с использованием двумерной инфракрасной спектроскопии было установлено, что ядерные движения тяжелой воды качественно отличаются от подобных движений в $\mathrm{H}_{2} \mathrm{O}$. Нелинейный спектр жидкости $\mathrm{D}_{2} \mathrm{O}$ показывает отличные от воды O-D резонансы. Это различие в молекулярной динамике отражает сдвиг баланса между внутримолекулярными и межмолекулярными водородными связями. В [3] структура $\mathrm{D}-\mathrm{O}$ и $\mathrm{H}_{2} \mathrm{O}$ исследована методом рамановской спектроскопии в диапазоне температур от 303 до $573 \mathrm{~K}$ и установлено, что $\mathrm{D}_{2} \mathrm{O}$ и $\mathrm{H}_{2} \mathrm{O}$ имеют одинаковые типы водородных связей, но различную их конфигурацию: O . . D - О более симметрична и сильнее на $\sim 20 \%$, чем $\mathrm{O} \ldots \mathrm{H}-\mathrm{O}$.

Вода, существующая в геологических, биологических и инженерных средах, часто присутствует в полостях нанометрового размера или адсорбируется в виде водяных пленок нанометрового размера (ограниченная вода). Также ограниченная вода распространена в биологических системах и мембранах, используемых для ее очистки. Пространственное ограничение приводит к отклонениям в термодинамических и физических свойствах воды по сравнению с наблюдаемыми в объемной неограниченной фазе [4-6]. Следовательно, существует необходимость понять влияние наноразмерного ограничения на физико-химические свойства такой воды. Для исследования ограниченной воды используются 4 различные модельные структуры: нанопоры, обратные мицеллы, органические растворители и гидратные оболочки (в частности, гидратные оболочки белков). Удержание воды наблюдается наиболее сильным в нанопорах, затем следуют обратные мицеллы, органические растворители и гидратирующие оболочки. В данной работе для исследования использовались пористые стекла (ПС) с радиусом пор от 2 до $10 \mathrm{~nm}$.

ПС - материал с уникальными свойствами. Он имеет поры нанометровых размеров, связанные друг с другом и образующие трехмерную сеть, обеспечивающую чрезвычайно большое отношение площади поверхности пор к их объему [7]. Поэтому этот материал имеет высокий потенциал для использования в качестве платформы для 
Таблица 1. Значения частот $v$ максимумов гауссовых компонент разложения ИК спектров $\mathrm{H}_{2} \mathrm{O}$ в ПС

\begin{tabular}{c|c|c|c|c|c|c|c}
\hline Номер пика & 1 & 2 & 3 & 4 & 5 & 6 & 7 \\
\hline$v, \mathrm{~cm}^{-1}$ & 3620 & 3490 & 3420 & 3360 & 3304 & 3240 & 3175
\end{tabular}

Таблица 2. Значения частот $v$ максимумов гауссовых компонент разложения ИК спектров $\mathrm{D}_{2} \mathrm{O}$ в ПС

\begin{tabular}{c|c|c|c|c|c|c|c}
\hline Номер пика & 1 & 2 & 3 & 4 & 5 & 6 & 7 \\
\hline$v, \mathrm{~cm}^{-1}$ & 2670 & 2607 & 2553 & 2498 & 2448 & 2383 & 2343
\end{tabular}

фиксации различных молекул. Большой свободный объем в таких структурах играет большую роль в процессах трансляционной и вращательной диффузии адсорбированных в порах молекул, поэтому ПС использовались для исследования поведения сложных молекул, в частности обладающих люминесцентной способностью $[8,9]$.

В представленной работе исследовано влияние нанопор ПС на свойства $\mathrm{H}_{2} \mathrm{O}$ и $\mathrm{D}_{2} \mathrm{O}$. Одним из перспективных методов таких исследований является метод „колебательного“ зонда, когда анализируются колебательные спектры (ИК и спектры комбинационного рассеяния) адсорбированных молекул $[6,10]$. Мы в качестве метода исследования применили ИК спектроскопию.

\section{Материалы и методы}

В качестве образцов использовались натриевоборосиликатные ПС с различными радиусами пор, изготовленные из стекол модифицированных составов на базе марки стекла ДВ-1, содержащего $\mathrm{SiO}_{2}, \mathrm{Na}_{2} \mathrm{O}, \mathrm{B}_{2} \mathrm{O}_{3}$ с незначительным количеством примесей. Описание методики приготовления таких ПС описано в [7].

Величина удельной поверхности образцов определялась адсорбционным методом на стандартном волюмометрическом оборудовании Sorptomatic 1990 фирмы Thermo Electron Corporation методом BET (Brunauer, Emmet, Teller) по адсорбции азота. Затем по десорбционной ветви изотермы в интервале относительных давлений от 0.3 до $0.95 P / P_{0}$ методом ВЈН (Barrett, Joyner, Halenda) было рассчитано распределение объема мезопор по размерам и средние размеры пор в образцах.

В качестве объектов исследования были выбраны бидистиллированная и дейтериевая вода $\left(99 \% \mathrm{D}_{2} \mathrm{O}\right.$ и $1 \% \mathrm{H}_{2} \mathrm{O}$ ), которые инфильтровались в ПС. ИК спектры регистрировали при комнатной температуре $\left(20^{\circ} \mathrm{C}\right)$ на фурье-спектрометре Varian 3100 FT-IR. Для уменьшения влияния поглощения ПС на результаты измерений ИК спектров $\mathrm{D}_{2} \mathrm{O}$ и $\mathrm{H}_{2} \mathrm{O}$ применялся метод нарушенного полного внутреннего отражения (НПВО).

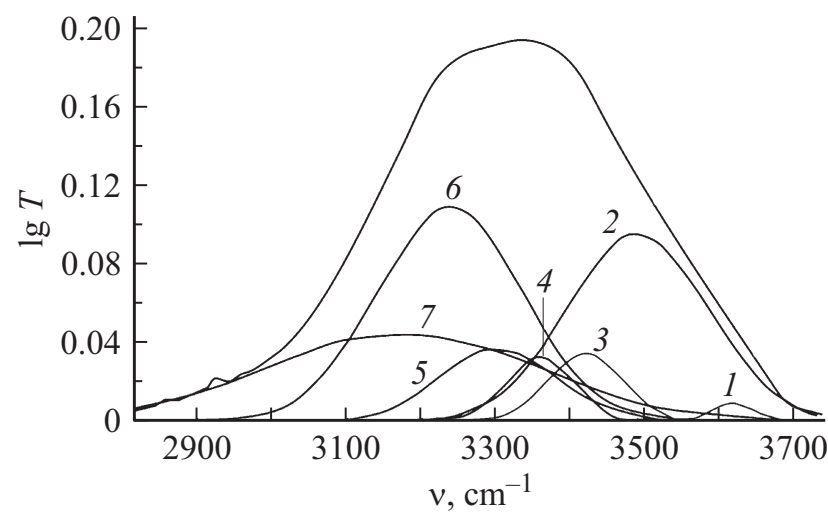

Рис. 1. ИК спектры поглощения $\mathrm{H}_{2} \mathrm{O}$ в ПС $(R=9.8 \mathrm{~nm})$ и разложение спектра на гауссовы компоненты.

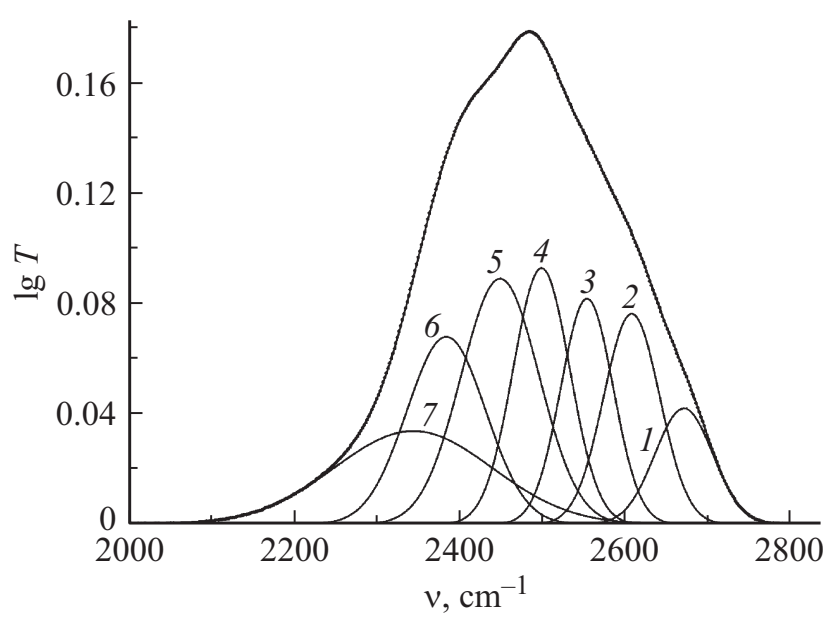

Рис. 2. ИК спектры поглощения $\mathrm{H}_{2} \mathrm{O}$ в ПС $(R=3.7 \mathrm{~nm})$ и разложение спектра на гауссовы компоненты.

\section{Результаты и обсуждение}

На рис. 1 и 2 представлены ИК спектры $\mathrm{H}_{2} \mathrm{O}$ и $\mathrm{D}_{2} \mathrm{O}$ в ПС. На этих же рисунках представлены их разделения на 7 гауссовых спектров. В табл. 1 и 2 указаны значения частот $v_{i}$ максимумов гауссовых компонент. Согласно $[11,12]$, низкочастотные гауссовы компоненты с $v_{7}=3175, v_{6}=3240, v_{5}=3405, v_{4}=3360$ и $v_{7}=3420 \mathrm{~cm}^{-1}$ для $\mathrm{H}_{2} \mathrm{O}$ и $v_{7}=2343, v_{6}=2383$, $v_{5}=2448, v_{4}=2498$ и $v_{7}=2553 \mathrm{~cm}^{-1}$ для $\mathrm{D}_{2} \mathrm{O}$ можно отнести к молекулам с сильной Н-связью. Пики $v_{1}=3620$ и $v_{2}=3490 \mathrm{~cm}^{-1}$ для $\mathrm{H}_{2} \mathrm{O}$ и $v_{1}=2670$ и $v_{2}=2607 \mathrm{~cm}^{-1}$ для $\mathrm{D}_{2} \mathrm{O}$ относятся к симметричным и антисимметричным растяжениям $\mathrm{H}$-слабо связанных молекул соответственно. В полосах, соответствующих сильно $\mathrm{H}$-связанных молекул $\mathrm{H}_{2} \mathrm{O}$ и $\mathrm{D}_{2} \mathrm{O}$ пики $v_{6}$ и $v_{5}$ обусловлены синфазными колебаниями всех молекул в совокупности, в то время как пики $v_{3}$ относятся к колебаниям, которые не являются синфазными между соседними молекулами. 


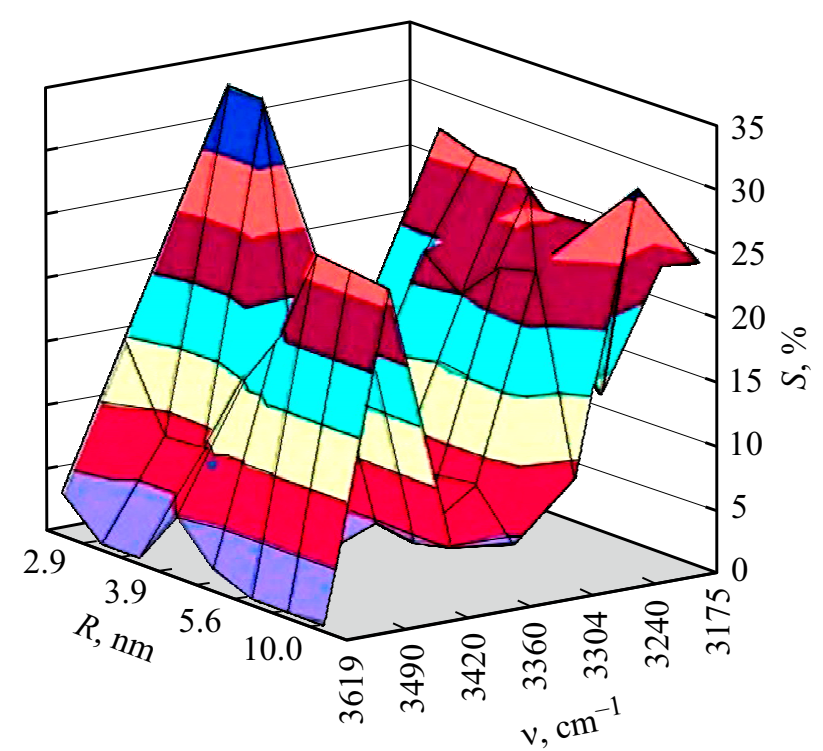

Рис. 3. Зависимость $S$ гауссовых компонент разложения ИК спектров $\mathrm{H}_{2} \mathrm{O}$ от частоты их максимума и $R$ пор.

Для анализа влияния размеров пор ПС на эффективность различных колебаний были определены площади $S$ гауссовых компонент для различных $R$. На рис. 3 представлена зависимость $S$ от $R$ и $v$ для $\mathrm{H}_{2} \mathrm{O}$. Из рис. 3 видно, что наиболее интенсивными являются колебания $v_{2}, v_{5}, v_{6}$ и $v_{7}$, наименьшей интенсивностью обладают полосы $v_{1}, v_{3}$ и $v_{4}$. Для полос $v_{1}, v_{4}$ и $v_{7}$ не наблюдается изменение $S$ в зависимости от $R$ пор. При этом вклад полос с $v_{1}$ в формировании ИК спектров поглощения незначительный $(\sim 2 \%)$, в то время как для $v_{7}$ он существен $(\sim 25 \%)$, для полосы $\nu_{4}$ составляет $\sim 7 \%$.

Изменение $S$ от $R$ наблюдается для полос $v_{2}, v_{3}$ и $v_{6}$. Для $R$ в диапазоне $1.85-2.2 \mathrm{~nm} S$ для $v_{3}$ сильно растет (на $\sim 20 \%$ ), для $v_{2}-$ уменьшается незначительно (на $\sim 6 \%$ ), для $v_{6}$ наблюдается небольшой рост (на $\sim 2 \%$ ). В области $2.2<R<3.2 \mathrm{~nm}$ изменение $S$ для всех трех частот практически не наблюдается. Для $3.2<R<3.7$ наблюдается резкое уменьшение $S$ для $v_{3}$ (на $\sim 27 \%$ ) и незначительное увеличение для $v_{2}$ $\left(\right.$ на $\sim 7 \%$ ) и $v_{6}$ (на $\left.\sim 10 \%\right)$. Для пор с $R>4$ наблюдается незначительный рост $S$ для $v_{2}$ и $v_{3}$ и уменьшение для $v_{6}$. Для ПС с $R>6$ изменения в гауссовых компонентах ИК спектров поглощения $\mathrm{H}_{2} \mathrm{O}$ не наблюдаются.

Для $\mathrm{D}_{2} \mathrm{O}$ зависимость $S$ от $R$ и $v$ представлена на рис. 4. Из рис. 4 видно, что в отличие от $\mathrm{H}_{2} \mathrm{O}$ для $\mathrm{D}_{2} \mathrm{O}$ наблюдается незначительное различие в $S$ для молекул с сильной Н-связью (низкочастотные гауссовы компоненты с $v_{7}, v_{4}, v_{5}, v_{6}$ и $\left.v_{7}\right)$ и слабой Н-связью (высокочастотные пики гауссовых компонент ИК спектра с $v_{1}$ и $\left.v_{2}\right)$. Так, для $\mathrm{D}_{2} \mathrm{O}$ суммарная интенсивность $S$ всех полос молекул с сильной Н-связью равна $\sim 15 \%$, в то время как для полос молекул со слабой Н-связью $\sim 7 \%$. Для $\mathrm{H}_{2} \mathrm{O}$ же $S$ для всех полос для молекул с сильной Н-связью равна $\sim 27 \%$, а для молекул со слабой Н-связью 4\%. Также наблюдается различие в интенсивностях $S$ для полос с симметричным $\left(v_{1}\right)$ и несимметричным $\left(v_{2}\right)$ растяжением слабой Н-связи: для $\mathrm{H}_{2} \mathrm{O}$ отношение $\frac{S_{v_{2}}}{S_{v_{1}}} \approx 3.5$, а для $\mathrm{D}_{2} \mathrm{O}-\frac{S_{v_{2}}}{S_{v_{1}}} \approx 1.85$.

Зависимость $S$ от $R$ для $\mathrm{D}_{2} \mathrm{O}$ также отличается от аналогичной зависимости для $\mathrm{H}_{2} \mathrm{O}$. Для частот $v_{5}, v_{4}$, $v_{3} S$ для разных $R$ практически одинакова. Изменение $S$ от $R$ наблюдается для $v_{1}, v_{6}$ и $v_{7}$ (рис. 4 ).

Для анализа влияния размеров пор на структуру $\mathrm{H}_{2} \mathrm{O}$ и $\mathrm{D}_{2} \mathrm{O}$ были вычислены отношения $A 1=\frac{S_{v_{7}}+S_{v_{6}}+S_{v_{5}}}{S_{v_{1}}+S_{v_{2}}}$ для различных значения $R$. A1 представляет собой отношение доли молекул воды с сильной Н-связью к доле Н-слабо связанных молекул воды.

На рис. 5 представлены зависимости $A 1$ для $\mathrm{H}_{2} \mathrm{O}$ (кривая 1) и для $\mathrm{D}_{2} \mathrm{O}$ (кривая 2) от радиуса пор $R$. Из рис. 5 видно, что для $\mathrm{H}_{2} \mathrm{O}$ наблюдается резкое умень-

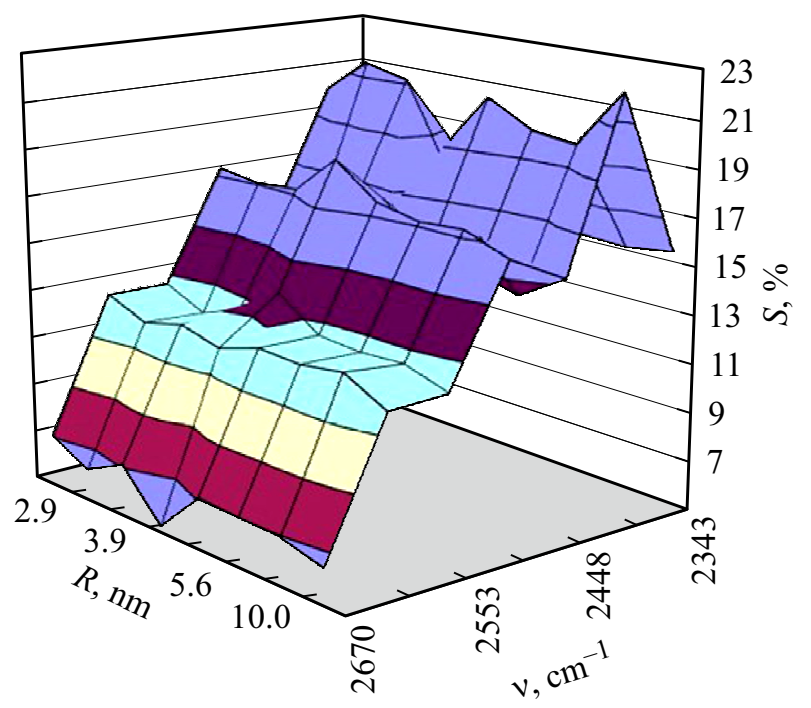

Рис. 4. Зависимость $S$ гауссовых компонент разложения ИК спектров $\mathrm{D}_{2} \mathrm{O}$ от частоты их максимума и $R$ пор.

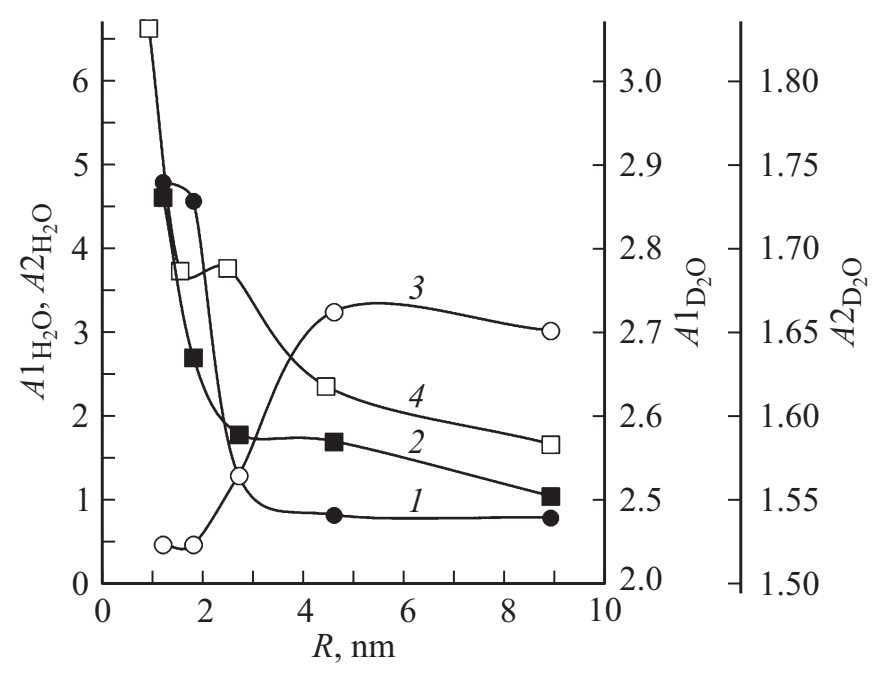

Рис. 5. Зависимости $A 1_{\mathrm{H}_{2} \mathrm{O}}(1), A 1_{\mathrm{D}_{2} \mathrm{O}} \quad(2), A 2_{\mathrm{H}_{2} \mathrm{O}}$ и $A 2_{\mathrm{D}_{2} \mathrm{O}}(4)$ от радиусов пор $R$ стекла. 
шение $A 1$ с ростом $R$ (с $\sim 5$ до 1$)$. То есть с ростом $R$ наблюдается разрыв водородных связей в $\mathrm{H}_{2} \mathrm{O}$. Связано это с тем, что молекулы воды в замкнутом пространстве собираются вокруг поверхностных гидроксильных групп с образованием островков высококоррелированных локализованных водных областей, за счет этих островков образуется прерывистая сеть Н-связей. Аналогичная зависимость $A 1$ от $R$ наблюдается и для $\mathrm{D}_{2} \mathrm{O}$, только изменение $A 1$ незначительно (с 1.73 до 1.55 , см. рис. 5 , дополнительная шкала). Это обусловлено тем, что хотя $\mathrm{D}_{2} \mathrm{O}$ имеет одинаковые с $\mathrm{H}_{2} \mathrm{O}$ типы водородных связей, но связь D-O более симметрична и более сильна [13].

Уменьшение числа водородных связей в $\mathrm{H}_{2} \mathrm{O}$ и $\mathrm{D}_{2} \mathrm{O}$ при увеличении $R$ сопровождается изменением соотношения $A 2=\frac{S_{v_{6}}}{S_{v_{3}}}$, которое характеризует отношение доли молекул с синфазными колебаниями всех молекул к доле молекул с несинфазными колебаниями между соседними молекулами. На рис. 5 представлены зависимости $A 2(R)$ для $\mathrm{H}_{2} \mathrm{O}$ (кривая 3) и $\mathrm{D}_{2} \mathrm{O}$ (кривая 4). Из рис. 5 видно, что для $\mathrm{H}_{2} \mathrm{O}$ в области $R \sim 3-6 \mathrm{~nm}$ с увеличением $R$ происходит значительное (с 0.5 до 3 ) увеличение $A 2$ (кривая 3). Это означает, что молекулы, которые образуют связи с поверхностью пор, в основном формируются из молекул сетки водородных связей, колебания которых происходят не синфазно. В противоположность этому для $\mathrm{D}_{2} \mathrm{O}$ наблюдается, хотя и незначительное (с 1.85 до 1.6) уменьшение параметра $A 2$ с увеличением $R$. То есть для $\mathrm{D}_{2} \mathrm{O}$ молекулы, которые образуют связи с поверхностью пор, в основном формируются из молекул сетки водородных связей, колебания которых происходят синфазно. При $R>6 \mathrm{~nm}$ как для $\mathrm{H}_{2} \mathrm{O}$, так и для $\mathrm{D}_{2} \mathrm{O} A 2$ не изменяется при увеличении $R$.

\section{Заключение}

$\mathrm{B}$ настоящей работе проведено детальное исследование ИК спектров $\mathrm{H}_{2} \mathrm{O}$ и $\mathrm{D}_{2} \mathrm{O}$ (в области $\mathrm{O}-\mathrm{H}$ и $\mathrm{D}-\mathrm{O}$ полос) в условиях пространственного ограничения в матрицах нанопористого стекла с различными значениями радиусов пор. Для интерпретации ИК спектров было применено их разделение на 7 гауссовых компонент, каждая их которых описывает конфигурацию $\mathrm{H}_{2} \mathrm{O}$ и $\mathrm{D}_{2} \mathrm{O}$. Установлено, что с увеличением размера пор ПС в области $2<R<6 \mathrm{~nm}$ происходит уменьшение доли воды с сильной Н-связью и увеличение доли воды со слабой Н-связью молекул. При $R>6 \mathrm{~nm}$ изменение в структуре геометрически ограниченной $\mathrm{H}_{2} \mathrm{O}$ и $\mathrm{D}_{2} \mathrm{O}$ не наблюдается.

Для $\mathrm{D}_{2} \mathrm{O}$ этот эффект меньше. Объясняется это тем, что связи $\mathrm{D}-\mathrm{O}$ сильнее, чем $\mathrm{H}-\mathrm{O}$. При этом молекулы $\mathrm{H}_{2} \mathrm{O}$, которые образуют связи с поверхностью пор, в основном формируются из молекул сетки водородных связей, колебания которых происходят не синфазно, в то время как для $\mathrm{D}_{2} \mathrm{O}$ такие связи с поверхностью формируются из молекул сетки водородных связей, колебания которых происходят синфазно.

\section{Конфликт интересов}

Авторы заявляют, что у них нет конфликта интересов.

\section{Список литературы}

[1] Защепина Г.Н. Свойства и структура воды. М.: Изд-во МГУ, 1974. $168 \mathrm{c}$.

[2] De Marco L., Carpenter W., Liu H., Biswas R., Bowman J.M., Tokmakoff A. // J. Phys. Chem. Lett. 2016. V. 7. N 10. P. 1769. doi 10.1021/acs.jpclett.6b00668

[3] Qingcheng Hu, Shunli Ouyang, Jie Li, Zhao Cao // J. Raman Spectrosc. 2017. V. 48. P. 610. doi 10.1002/jrs.5088

[4] Yuzi He, Ken-ichi Nomura, Rajiv K. Kalia, Aiichiro Nakano, Priya Vashishta // Phys. Rev. Mater. 2018. V. 2. P. 115605. doi 10.1103/PhysRevMaterials.2.115605

[5] Levitz P. // Molecular Physics. 2019. V. 117. N7-8. P. 952. doi 10.1080/00268976.2018.1527960

[6] Knight A.W., Kalugin N.G., Coker E., Ilgen A.G. // Scientific Reports. 2019. V. 9. P. 8246. doi 10.1038/s41598-019-44651-z

[7] Пак В.Н., Гавронская Ю.Ю., Буркат Т.М. Пористые стекла и наноструктурированные материалы на их основе. СПб.: Изд-во РГПУ им. А.И. Герцена, 2013.

[8] Afanasyev D.A., Ibrayev N.Kh., Saletsky A.M., et al. // J. Luminescence. 2013. V. 136. P. 358 doi 10.1016/j.jlumin.2012.11.013

[9] Старокуров Ю.В., Летута С.Н., Пашкевич С.Н., Антропова Т.В., Гордеева Ю.А., Салецкий А.М. // Опт. и спектр. 2013. T. 114. N 1. C. 95. doi 10.7868/S003040341301025X; Starokurov Y.V., Gordeeva Y.A., Saletsky A.M., Letuta S.N., Pashkevich S.N., Antropova T.V. // Opt. Spectrosc. 2013. V. 114. N 1. P. 87. doi 10.1134/S0030400X13010256

[10] Горелик В.С., Ві Dongхие, Войнов Ю.П., Водчии, А.И., Орлович В.А., Савельева А.И. // Опт. и спектр. 2019. Т. 126. N 6. 765. doi 10.21883/OS.2019.06.47771.51-19; Gorelik V.S., Dongxue Bi, Voinov Yu.P., Vodchits A.I., Orlovich V.A., Savel'eva A.I. // Opt. Spectrosc. 2019. V. 126. N 6. P. 687. doi 10.1134/S0030400X19060079

[11] Walrafen G.E., Fisher M.R., Hokmabadi M.S., Yang W.-H. // J. Chem. Phys. 1986. V. 85. P. 6970. doi 10.1063/1.451384

[12] Green J.L., Lacey A.R., Sceats M.G. // Phys. Chem. 1986. V. 90 . N 17. P. 3958. doi $10.1021 / \mathrm{j} 100408 \mathrm{a} 027$

[13] Qingcheng Hu, Shunli Ouyang, Jie Li, Zhao Cao // J. Raman Spectrosc. 2017. V. 48. P. 610. doi 10.1002/jrs.5088 